Supporting Information

\title{
Induced Self-aspiration Electrospray Ionization Mass Spectrometry for Flexible Sampling and Analysis
}

\author{
Qian Zhang ${ }^{1,2}$, Yanping Zhu ${ }^{1}$, Yuan Tian ${ }^{1}$, Quan $\mathrm{Yu}^{1, *}$, Xiaohao Wang ${ }^{1,2}$ \\ 1 (Division of Advanced Manufacturing, Tsinghua Shenzhen International Graduate School, Shenzhen, \\ 518055) \\ 2 (State Key Laboratory of Precision Measurement Technology and Instruments, Department of Precision \\ Instruments and Mechanology, Tsinghua University, Beijing 100084) \\ * Correspondence to: yu.quan@sz.tsinghua.edu.cn
}




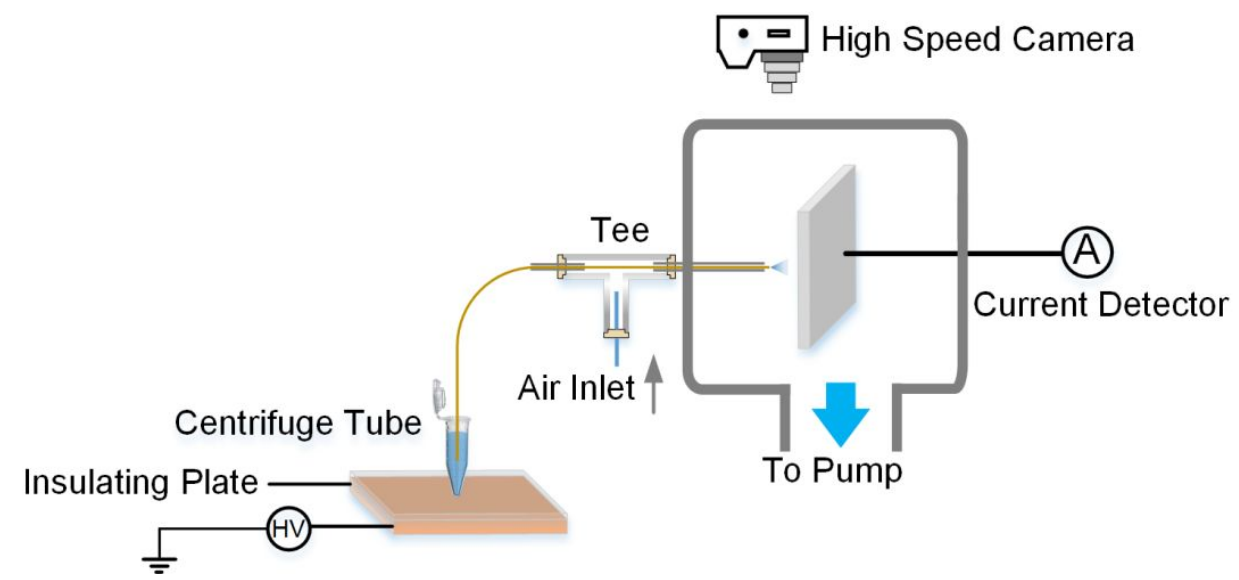

Figure S1. Schematic of the test platform constructed to characterize ISA-ESI.

In order to better characterize the ISA-ESI process, a sub-ambient pressure chamber with an ion current detector and a high-speed camera was specially constructed. A miniature diaphragm pump (MVP 006, Pfeiffer Vacuum Inc.) was used to maintain the chamber pressure at $70 \mathrm{kPa}$. As shown in Figure S1, the outlet of the ISA-ESI source was placed in the chamber, $5 \mathrm{~mm}$ from the ion detection plate. The acquired ion current was detected by a sensitive current detector (KEITHLEY 6430, Tektronix). The top cover of the chamber was made of transparent glass, above which a high-speed camera (Phantom VEO 710) was installed to capture the spray process. 

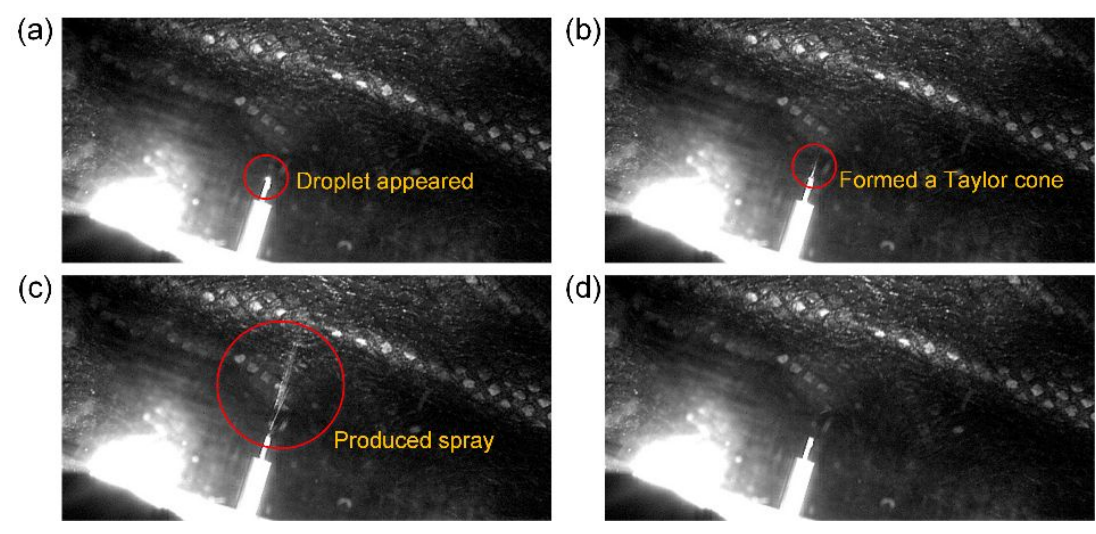

Figure S2. Different stages of a typical ISA-ESI cycle captured by the high-speed camera: (a) charged liquid reaching capillary outlet; (b) forming a Taylor cone; (c) fully electrospraying; (d) end of spray. Digital camera sampled rate was set at $200 \mathrm{fps}$ and the resolution was $512 * 256$.

A methanol solution containing $1 \%$ formic acid was used as the spray solvent, and a $7 \mathrm{kV}$ voltage was applied on the electrode plate. For the ISA-ESI operation, the injection time was set at $500 \mathrm{~ms}$ and the interval between each injection was $20 \mathrm{~s}$. A few seconds after the solution was pulsed sampled, an electrospray was formed at the capillary outlet. This process was recorded by the high-speed camera (sampling rate of $200 \mathrm{fps}$ and the resolution was 512*256.) and shown in Figure S2. Herein, the formation of the Taylor cone (Figure S2b) and electrospray (Figure S2c) could be clearly observed. In addition, there were no residual droplets remaining in the capillary outlet after spraying. 

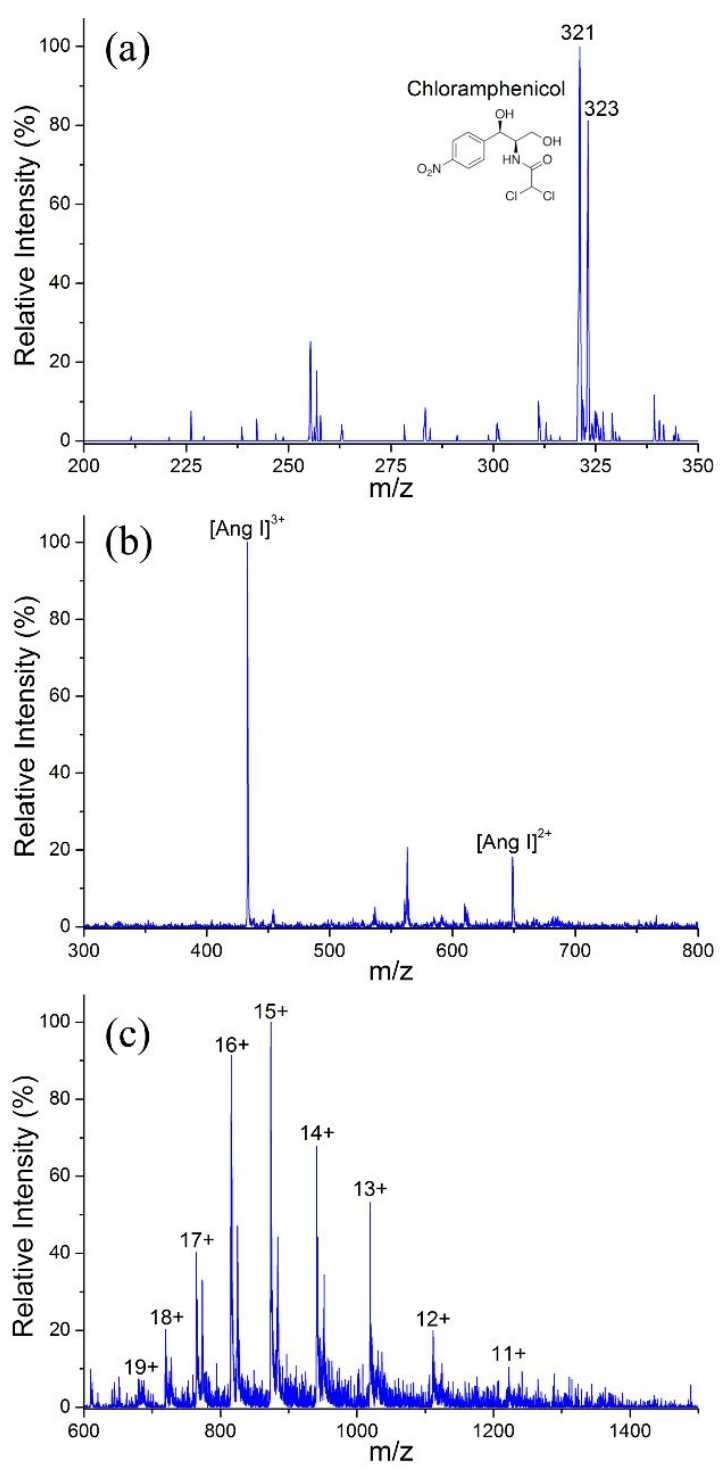

Figure S3. Typical ISA-ESI mass spectrum of (a) chloramphenicol acquired in negative MS mode, (b) angiotensin I and (c) cytochrome C obtained in positive MS mode.

ISA-ESI will only produce ions with a single polarity, which was decided by the HV applied on the electrode plate. The spectrum of chloramphenicol given in Figure S3a was acquired at negative MS mode with $-5 \mathrm{kV}$ voltage applied, while the ISA-ESI spectra of angiotensin (Figure S3b) and cytochrome C (Figure S3c) were obtained using positive MS mode. These results indicated that the ionization capability of ISA-ESI was similar to conventional ESI. 

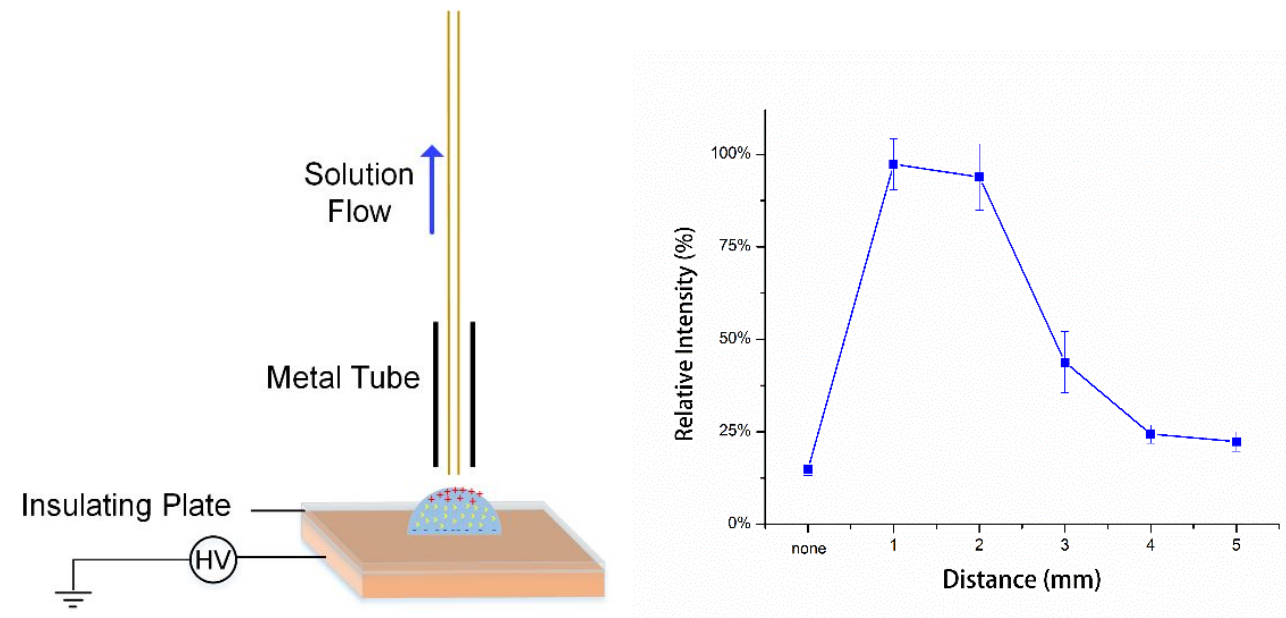

Figure S4. (a) schematic of the optimized ISA-ESI structure with a metal tube; (b) relative ion intensity acquired at different metal tube positions.

Based on the capacitive coupling effect, electrospray can be generated with no physical contact between the electrode and the solution. In ISA-ESI, the efficiency of electrospray is related to the amount of charge accumulated in the liquid column. According to the definition formula of the capacitor $\mathrm{C}=\mathrm{Q} / \mathrm{U}(\mathrm{C}$ is the capacitance, $\mathrm{Q}$ is the charge amount in the capacitor, $\mathrm{U}$ is the applied voltage), increase $\mathrm{C}$ at a constant $\mathrm{U}$ can increase Q. Hence, as shown in Figure S4(a), a metal tube was mounted outside the capillary as the counter electrode to increase the capacitance of the electrode-capillarysolution system. In this case, more charges can be accumulated in the solution. The modified ISA-ESI source has been tested and characterized by analyzing a $10 \mathrm{ppm}$ rotundine solution. The position of the metal tube should be carefully regulated because it can affect the charged capacity of the solution. By changing the distance from the metal tube end to the liquid surface, the total ion current signal intensity acquired can significantly change, as illustrated in Figure S4b. It seems shortening the distance between the metal tube and the solution can facilitate the charge accumulation in the liquid. However, it may also increase the possibility of discharge between the tube and the solution during sampling. 


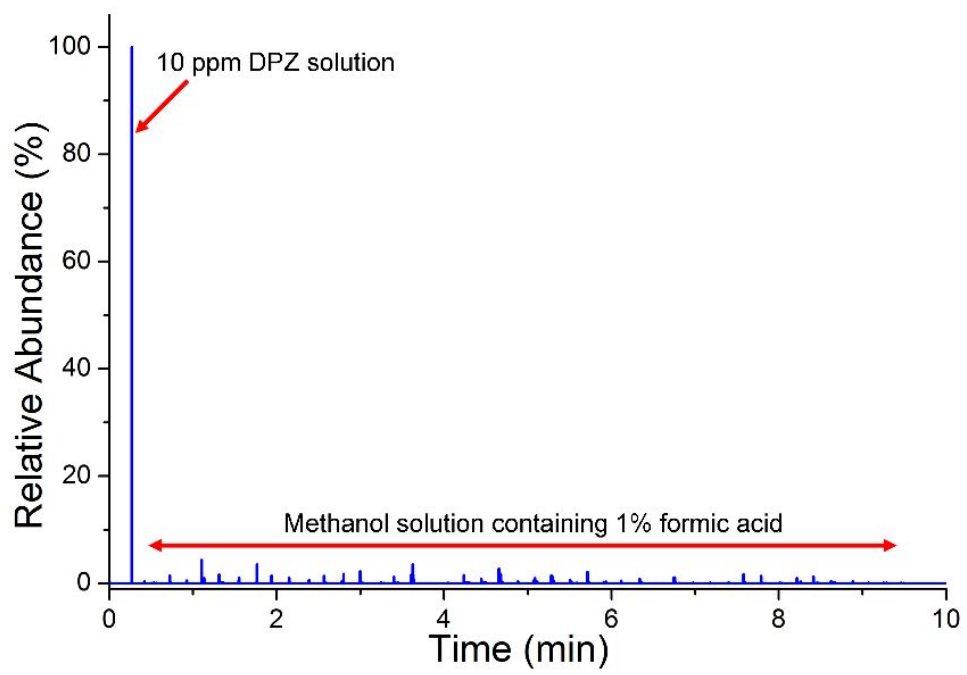

Figure S5. Extracted ion current of dioxoprozine hydrochloride $(\mathrm{m} / \mathrm{z} 317)$ acquired in a cleaning step. A methanol solution containing 1\% formic acid and $10 \mathrm{ppm}$ dioxoprozine hydrochloride was sampled first, and then a pure solvent (methanol solution containing $1 \%$ formic acid) was used for cleaning. It can be seen that relatively low DPZ ion signal (less than $5 \%$ ) is observed in the whole cleaning process, indicating that the solvent can effectively remove the residual.

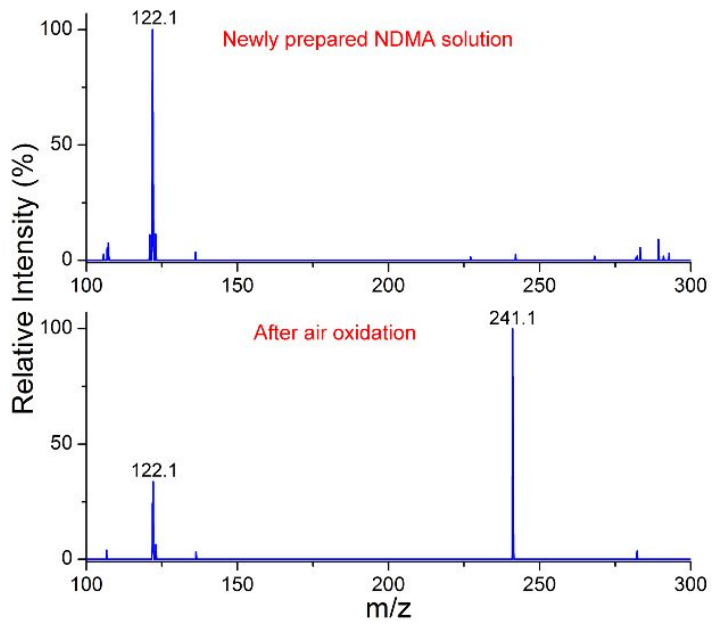

Figure S6. ISA-ESI-MS spectra of a newly prepared NDMA solution and a sufficiently irradiated NDMA solution. Both solutions were shaken before analysis. Only the NDMA ions $(\mathrm{m} / \mathrm{z} 121)$ but no product ions $(\mathrm{m} / \mathrm{z} 241)$ appeared in the spectrum of the new NDMA solution. In contrast, a large number of product ions of the oxidation reaction can be observed in the irradiated solution. 


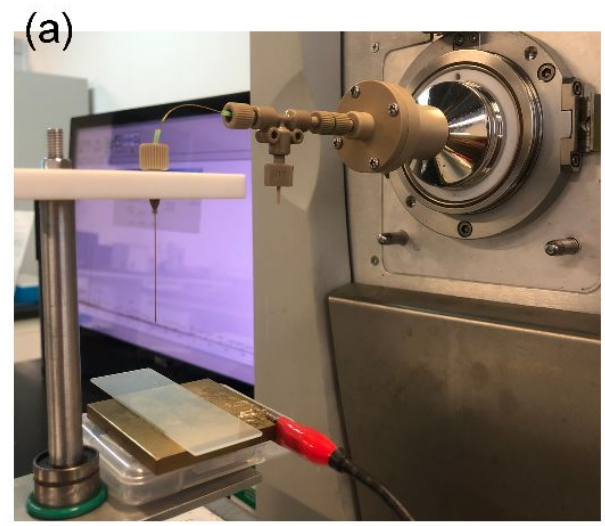

(b)

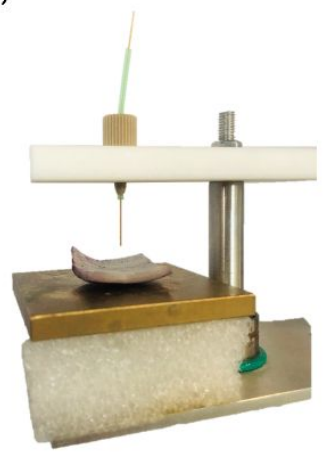

Figure S7. (a) Photograph of the developed ISA-ESI source and (b) it is being used in LESA analysis of onion. 